\title{
Cases with parietal and occipital lobe epilepsies
}

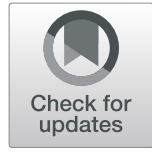

\author{
Hermann Stefan
}

\begin{abstract}
Background: For seizures emerging from the posterior cortex it can be a challenge to differentiate if they belong to temporal, parietal or occipital epilepsies. Sensoric auras like visual phenomena may occur in all of these focal epilepsies. Ictal signs may mimic non- epileptic seizures.

Case presentations: Case 1: Patient suffering from a pharmacoresistent focal epilepsy. Focal seizures with sudden visual disturbance, later during the seizure epigastric aura, vertigo-nausea, involvement to bilateral tonic-clonic seizures. MEG detected interictal spikes, source localization indicated focal epileptic activity parietal right.

Case 2: Patient with focal pharmacoresistent epilepsy, semiology with focal unaware seizures, feeling that something like a coat is imposed from behind on him, then feeling cold over the whole body, goose bumbs from both arms to head, then block of motoric activity, later focal unaware seizures with stare gaze, blinking of eyes, clouding of consciousness, elevation of arms and legs, sometimes tonic-clonic convulsions. EEG/MEG source localization and MRI detected an epileptogenic lesion parietal left.

Case 3: Patient with pharmacoresistent focal epilepsy, focal aware seizures, a dark spot occurring in the left visual field, sometimes anxiety during seizures (leading to the suspicion of non-epileptic psychogenic pseudo seizures). MRI demonstrated an atrophy occipito-temporal right after sinus vein thrombosis. Ictal video-EEG showed a focal seizure onset occipital right.

Conclusion: Contribution of noninvasive and/or invasive confirmation of the localization of the underlying focal epileptic activity in posterior cortex is illustrated. Characteristics of posterior cortex epilepsies are ventilated.
\end{abstract}

Keywords: Parietal-occipital lobe epilepsy, Posterior cortex, Diagnosis and treatment

\section{Introduction}

For seizures emerging from the posterior cortex it can be a challenge to differentiate whether they belong to temporal, parietal or occipital epilepsies. Sensoric auras like visual phenomena may occur in all of these focal epilepsies. Ictal signs may mimic non- epileptic seizures. For the localization of focal epileptic activity in addition to detailed analysis of the ictal semiology by video-EEG, structural imaging by means of MRI, high resolution electrophysiological non-invasive source imaging, positron emission tomography or single photon emission tomography can provide important information in difficult cases.The clinical findings of three cases are reported and discussed.

\section{Cases}

Case 1

The female patient 37 years of age suffered since her 3rd year of life from a pharmacoresistent focal epilepsy with different seizure types. Focal aware seizures with sudden visual disturbance, semiology later during the seizure epigastric aura, vertigo-nausea, headache temple right, duration 1-4 min, frequency 12 times per month, focal unaware seizures with or without stare gaze, oral automatisms, no reaction, memory impairment, in $50 \%$ of these seizures involvement to bilateral tonic-clonic seizures occurred.

Neurological examination was normal.

EEG showed spikes temporal-occipital right sided more often than fronto-temporal right.

(C) The Author(s). 2019 Open Access This article is distributed under the terms of the Creative Commons Attribution 4.0 International License (http://creativecommons.org/licenses/by/4.0/), which permits unrestricted use, distribution, and reproduction in any medium, provided you give appropriate credit to the original author(s) and the source, provide a link to the Creative Commons license, and indicate if changes were made. The Creative Commons Public Domain Dedication waiver (http://creativecommons.org/publicdomain/zero/1.0/) applies to the data made available in this article, unless otherwise stated. 
$M R I$ findings let suggest hippocampal sclerosis right. $M E G$ detected interictal spikes, source localization indicated focal epileptic activity parietal right (Fig. 1a, b + c).

\section{Case 2}

A male patient, age 37 years old, focal epilepsy since 7th year of life, pharmacoresistent.

Semiology: focal unaware seizures: feeling that something like a coat is imposed from behind on him, then feeling of cold over the whole body, goose bumbs from both arms to head, then block of motoric activity, later focal unaware seizures with stare gaze, blinking of eyes, clouding of consciousness, elevation of arms and legs, duration $15-30 \mathrm{~s}$, frequency 3-6 times per month, sometimes tonic-clonic convulsions.

Neurological examination was normal.

$E E G / M E G$ : Source localization during interictal spikes showed a cluster of dipoles parietal left.

MRI detected a lesion parietal left (Fig. 2).

\section{Case 3}

Fifteen years old female patient, suffering from seizures since her 7th years of life, pharmacoresistent focal epilepsy, in childhood leukemia, sinus vein thrombosis.
Semiology: Focal aware seizures: a dark spot occurring in the left visual field, sometimes anxiety during seizures (leading to the suspicion of non-epileptic psychogenic pseudoseizures), duration: seconds to minutes, frequency: several times per day.

Neurological examination and surface EEG were normal. During video EEG recording the patient reported seeing a "spot" in the visual field left. A seizure occurred. Simultaneously while seeing a spot in the left visual field in the ictal EEG seizure onset occurred occipital right (Fig. 3).

MRI demonstrated an atrophy occipito-temporal right after sinus vein thrombosis (Fig. 4).

\section{Discussion}

\section{Comment to case 1}

The EEG shows rapid propagation from parietal to temporal lobe. Vertigo provides a semiological hint for extratemporal seizure onset. The other ictal signs could lead to the wrong diagnosis of temporal lobe epilepsy. Non-invasive source localization by means of MEG/EEG strengthens the semiology based hypothesis of parietal focus with propagation to temporal and was used for guidance of invasive electrode implantation.

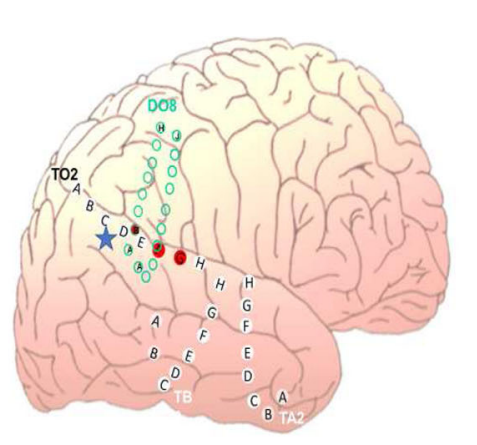

a

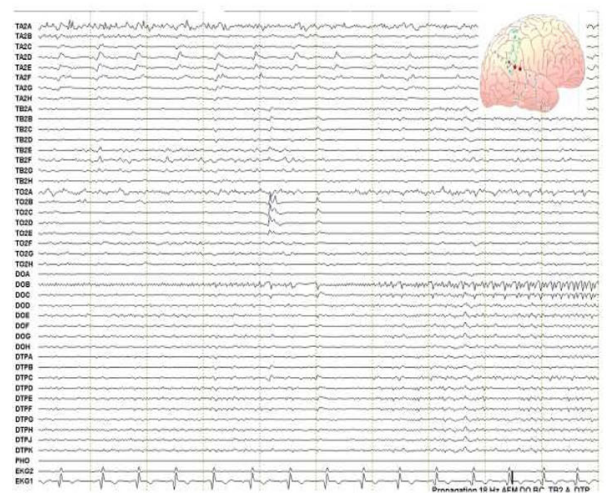

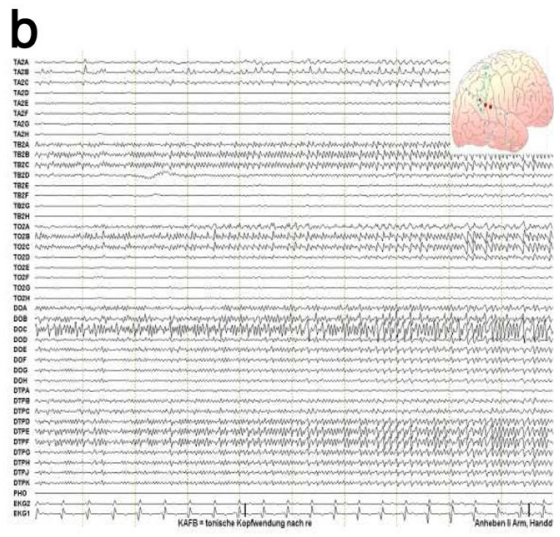

C

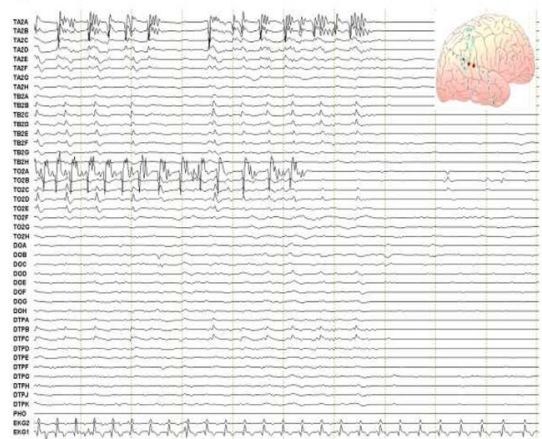

Fig. 1 Invasive electrodes, red seizure onset electrode contacts. MEG localization and different epochs during the seizure. Red: seizure onset in invasive electrodes a Ictal onset of seizure discharges parietal right. b + c Propagation to temporal anterior 




Fig. 2 Parietal left lesion identified as FCD2

\section{Comment to case 2}

The aura can be interpreted as related to a kind of "out of body experience", being localized to the parietal lobe. MRI and MEG confirmed the localization of epileptic activity in this area. In case of normal MRI postprocessing with morphometric analysis program (MAP) enables in some patients to detect "hidden" FCD II lesions in MRI. In this patient MRI showed the lesion already without MAP.

\section{Comment to case 3}

MRI showed a widespread atrophy after sinus vein thrombosis only. The clinical phenomenology suggested posterior cortex epilepsy. Anxiety during the seizure raised the question of non-epileptic attacks. Temporal lobe epilepsy or non-epileptic panic attacks were ruled out by ictal video-EEG recordings demonstrating a focal seizure onset in the occipital lobe during seizure onset with following propagation.

Compared to temporal or frontal lobe epilepsies parietal (PLO) and occipital lobe (OLE) epilepsies occur rarely. The occurrence for PLO is reported wih 5-8\% und for OLE 1.5-8\% [1-6]. Parietal (PLO) and occipital lobe(OLE) epilepsies are condensed as epilepsy of the posterior cortex.

Case 1 and 2 are diagnosed as parietal lobe epilepsies. In addition to the presentation of cases with clinical findings, characteristics of parietal epilepsies concerning ictal semiology, electrophysiology and imaging are discussed.

a) Seizure semiology

Most often focal aware seizures with somatosensoric ictal signs are registered. Epileptic discharges may arise from one of the three sensoric brain regions in the primary, secondary or insular sensory region. Somatosensory signs can occur contralateral to the seizure onset zone as well as ipsilateral or bilateral. They can be manifested as paresthesia, pain, temperature, sexual sensations and body scheme disturbances. Typical ictal signs are tingling, sensation of an electric blow. Body scheme disturbances can be manifested as changes of extremity shape even loss of extremity or other body parts. Other ictal signs may be ictal aphasia or anosognosia, acalculia, alexia, dysphasia, aphemia, vertigo or gustatory hallucinations. Motoric phenomena are more rare and more discrete (e.g. epileptic nystagmus). The patients may at least to some extent react to stimuli and recollect ictal signs.

During epileptic nystagmus the fast component beats to the side contralateral to the focus. Parietal cortical stimulation

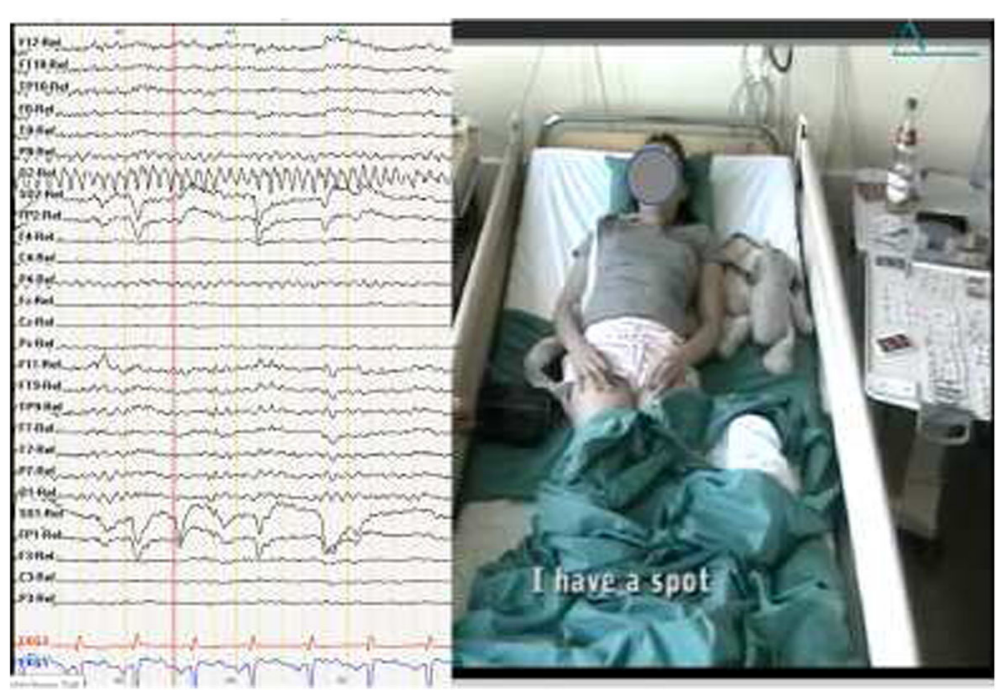

Fig. 3 Ictal onset in surface EEG occipital right. Simultaneously the patient reports seeing a "spot" in the visual field left 


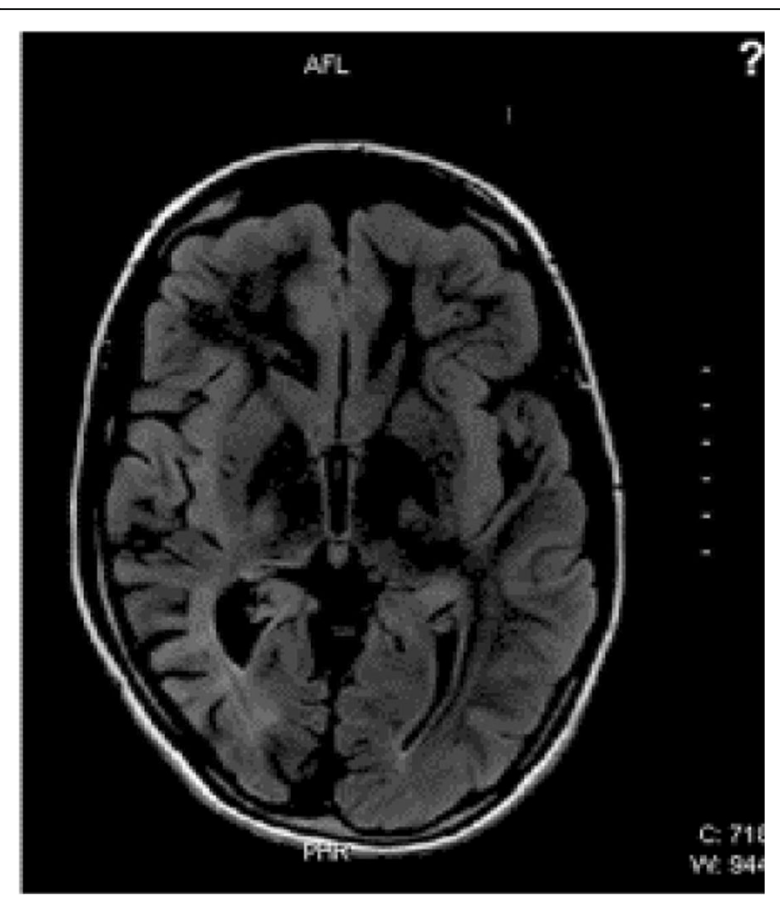

Fig. $4 \mathrm{MRI}$ after sinus vein thrombosis and atrophy posterior cortex right

during stereo-EEG-registration showed that the body scheme disturbances were only triggered in the non-dominant hemisphere whereas sensoric illusions or hallucinations as well as eyes/eyelid movements or sensations significantly cohered with the stimulation of the intraparietal sulcus [7]. Somatosensory sensations including motoric symptoms und dysarthria were detectable at stimulation of the postcentral gyrus. The "second sensory area", the left parietal opercula is involved in the supralaryngeal control. Ictal extremity agnosia and phantom extremity arise in der posterior parietal brain region. The neglect more often is associated with a right excitation of the inferior parietal brain. "Out-of-body experiences" are due to parieto-temporal excitations [8].

Because during parietal lobe seizures occur bilateral somatosensoric illusions combined with vertigo, disturbances of body scheme and only mild motoric signs (tremor, dyspractic movements), but with prominent emotional components such as fear or panic can be mistaken as psychogenic nonepileptic seizures.

\section{b) EEG}

During focal unaware seizures the surface EEG may show no unambiguous epileptiform activity. Because of rapid propagation surface EEG in many patients is not sufficient for localization of the focal epileptic activity. Source localization by means of MEG/EEG can provide improved information. In case of FCD type II related epilepsy MEG showed excellent surgical outcomes [9]. When MRI turns out as normal then propagated epileptic activity in a late phase of a seizure may be wrongly diagnosed as seizure onset region. Propagation is found frequently from parietal to temporal or frontal brain regions [3]. An invasive study was performed in 37 of the 38 patients for epilepsy surgery [10]. Seizure disappeared in 15 (Engel's classification I, 39.5\%), and rare seizure remained in 5 (Engel II, 13.2\%). Thirteen patients showed a worthwhile improvement (Engel III, 34.2\%), whereas 5 exhibited no worthwhile improvement (Engel IV, $13.2 \%)$.

\section{c) Imaging}

MRI showed abnormal findings in 68\%. MRI detected in $36.8 \%$ parietal lesions (28.95\% unilateral), hippocampal atrophy in $10.5 \%$, other atrophies $10.5 \%$ [10]. Most common cortical dysplasia was detected by histology in these operated patients. Of 39 patients seizures disappeared postoperatively in $39.5 \%$ (Engel class I) and rare seizure remained in $13.2 \%$ (Engel class II). $34.2 \%$ of the patients showed a worthwhile improvement (Engel class III), whereas $13.2 \%$ exhibited no worthwhile improvement (Engel class IV).

A meta-analysis of 253 operated patients (postoperative follow up $104.9+74.8$ months) showed Engel Class 1 in $62.4 \%$ [11].

\section{d) Etiology}

Histopathological findings of pharmacoresistent parietal lobe epilepsies were collected at the neuropathology reference center for epilepsy surgery Erlangen. In the resected brain tissue specimens after epilepsy surgery of 126 patients the following lesions were detected by histopathological examination: FCD II 17.8\%, glial scar 14.1\%, diffuse glioma $13.3 \%$, ganglioglioma $12.6 \%$, DNT 9.6\%, FCD not otherwise specified (NOS) 9.6\%), cavernoma 6.7\%, FCD1 6.7\%, no lesion $8.3 \%$, vascular malformation $4.4 \%$ [12].

Case 3 was diagnosed as occipital lobe epilepsy. Characteristic findings of occipital lobe epilepsies are as follows.

a) Seizure semiology

Ictal clinical signs are mainly visual and oculomotor. Often elementary visual hallucinations and postictal headache exist.

Visual phenomena build a functional link between parietal occipital lobe epilepsy: epileptic discharges from parieto-occipital transition region or occipital brain region may produce ictal visual phenomena. Elementary visual hallucinations are simple light sensations, colored spot, ring, occasionally with transient amaurosis contralateral to the seizure onset. Visual illusions can also be simply colour changes of objects.

Visual illusion or loss of vision not only exist in occipital lobe epilepsies, but also in occipito-temporal or even antero-temporal seizures. Complex hallucinations were not observed in occipital lobe patients [13]. Motoric ictal signs are lid blinking and contralateral eye movements. For visual processing two streams are differentiated: 
1. the dorsal stream via retina-pulvinar-posterior parietal cortex for recognition of object position

2. the ventral stream for the perception and object recognition via retina-primary visual cortex - inferior temporal cortex for the interpretation of the quality of an object and meaning. Visual phenomena usually are the first ictal sign of an occipital lobe seizure.

Fast propagation to frontal (30\%) or temporal (30\%) can overshadow it $[3,4]$. In case of reagibility of the patient this speaks in favour of onset in the calcarine area, if not then for onset in the temporo-occipital area. Ictal signs don't permit the definite differentiation of mesial or lateral occipital seizure onset [14].

The differential diagnosis of idiopathic occipital childhood epilepsy concerns mainly symptomatic occipital epilepsy and migraine. Elementary visual hallucinations of occipital seizures in idiopathic epilepsy develop rapidly within seconds, are brief in duration (2-3 min), frequent, usually colored and circular, whereas in case of migraine an aura slowly arises lasting $\geq 5 \mathrm{~min}$ and is mostly uncolored with linear shapes. In the Panayiotopoulus syndrome seizures occur with autonomic signs, the duration is longer and if visual symptoms exist they are not the only ictal manifestation.

b) Imaging

MRI detects like in parietal lobe epilepsy structural changes and provides important information for the localization hypothesis of the epileptogenic area.

cortical dysplasia 5/26, cerebromalacia 5/26, DNET, ganglioglioma calcification, infarction each $1 / 26$ in the epilepsy surgery report from Sang Kun Lee et al. 2005 [15]. MRI correctly localized the lesion in seven of 16 $(43.7 \%)$ postoperative seizure-free patients, FDG-PET in $50 \%$, ictal SPECT in $25 \%$.

c) EEG

EEG interictally shows unilateral posterior EEG slowing more common than occipital spikes.

In photosensitive OLE during intermittent photic stimulation spikes/polyspikes occipital or generalized spikes/ polyspikes with posterior accentuation are recorded [5]. The interictal EEG often shows spikes which rarely are correctly localized in the occipital lobe, but more often posterior temporal. In the ictal EEG epileptiform pattern may be missed or mislead the localization of seizure onset due to propagation to bilateral occipital or temporal regions. Source localization in MEG/EEG combined with MRI (MSI) are helpful for implantations of invasive electrodes.

Epilepsy surgery results are reported only in small numbers. Postoperative seizure free were $61.5 \%$ in 26 operated patients [15].

d) Etiology

Histopathological findings of patients with operated pharmacoresistent occipital lobe epilepsies showed in the resected brain tissue specimens similar lesion as in the parietal lobe epilepsies.

\section{Conclusion}

After describing the clinical findings concerning semiology, electrophysiology and imaging of these patients the differentiation from non-epileptic attacks and the localization of the posterior cortex for the seizure onset region is discussed. The contribution of noninvasive and/or invasive confirmation of the localization of the underlying focal epileptic activity is illustrated. Characteristics of posterior cortex epilepsies are ventilated.

Parietal and occipital lobe epilepsies represent a challenge for diagnosis and treatment. The combined view to ictal semiology, imaging, noninvasive source imaging and invasive recordings provide improved chances for correct diagnosis.

\section{Abbreviations}

DNT/ DNET: Dysembrioplastic neuroectodermal tumor; EEG: Electroencephalography; FCD: Focal cortical dysplasia; FDG-PET: Positron emission tomography; MAP: Morphometric analysis program; MEG: Magnetoencephalography; MRI/MSI: Magnetic resonance imaging; NOS: Not otherwise specified; OLE: Occipital lobe epilepsy; PLO: Parietal lob epilepsy; SPECT: Single photon emission computed tomography

\section{Acknowledgements}

Not applicable for that section.

\section{Disclosures}

H. Stefan received DFG grants, honoraria and travel reimbursement from Elekta Oy, Helsinki, Finland. There is no conflict of interest concerning the content of this paper.

Authors' contributions

The author read and approved the final manuscript.

Authors' information

Prof. Hermann Stefan is the sole author of this paper.

Funding

Not applicable for that section.

Availability of data and materials

Department of Neurology - Epilepsy Center, University Hospital Erlangen.

Ethics approval and consent to participate

Not applicable for that section.

Consent for publication

Not applicable for that section.

\section{Competing interests}

The author declares that he has no competing interests.

Received: 10 March 2019 Accepted: 6 August 2019

Published online: 18 September 2019

\section{References}

1. Sweinbornsdottier S, Duncan J. Parietal and occipital lobe epilepsy. Epilepsia. 1993;34:493-521.

2. Rasmussen $T$. Surgery for epilepsy arising in other regions than the temporal or frontal lobe. In: Purpura D, Penry J, Walter D, editors. Neurosurgical management of the epilepsies. New York: Raven Press; 1975. 
3. Williamson PD, Thadani VM, Darcey TM, et al. Occipital lobe epilepsy: clinical characteristics, seizure spread patterns, and results of surgery. Ann Neurol. 1992;31:3-13.

4. Salanova V. Parietal lobe epilepsy. J Clin Neurophysiol. 2012;29(5):392-6.

5. Adcock JE, Panayatopoulus CP. Occipital lobe seizures and epilepsies. Clin Neurophysiol. 2012;29(5):397-407.

6. Kasper B, Stefan H. Blind but able to see in Case studies in epilepsy. In: Stefan H, Menachem EB, Chauvel P, Guerrini R, editors. Cambridge Medicine; 2012. p. 104-7.

7. Balestrini $\mathrm{S}$, Francione S, Mai R, et al. Multimodal responses induced by cortical stimulation of the parietal lobe: a stereo -electroencephalography study. Brain. 2015;138(9):2596-607.

8. Mohr C, Blanke O. Illusory perceptions of the human body. Curr Psychiatry Rep. 2005;7:189-95.

9. Kasper BS, Rossler K, Hamer H, et al. Coregistrating magnetic source and magnetic resonance imaging for epilepsy surgery in focal cortical dysplasia. Neuroimage Clin. 2018;19:487-96.

10. Kim CH, Chung CH, Lee SK, Lee YK. Parietal lobe epilepsy:surgery and outcome. Stereotact Funct Neurosurgery. 2004;82(4):175-85.

11. Pilipovic-Dragovic S, Ristic A, Bukumiric Z, Trajkoc G, Sokic D. Long-term seizure outcome following epilepsy surgery in the parietal lobe: a metaanalysis. Epileptic Disord. 2018;20(2):116-22.

12. Blümcke I, Sarnat H, Coras R. Surgical neuropathology of focal epilepsies, John Libbey; 2015.

13. Bien $C$, Benninger $F$, Urback $H$, et al. Localizing value of epileptic visual auras. Brain. 2015;123(2):244-53.

14. Blume WT, Wiebe S, Tapsell LM. Occipital epilepsy: lateral versus mesial. Brain. 2005:128:1209-25.

15. Lee SK, Lee SY, Lee DS, Chung C-K. Occipital lobe epilepsies:clinical characteristics, surgical outcome and role of diagnostic modalities. Epilepsia. 2005:46(5):688-95.

Ready to submit your research? Choose BMC and benefit from:

- fast, convenient online submission

- thorough peer review by experienced researchers in your field

- rapid publication on acceptance

- support for research data, including large and complex data types

- gold Open Access which fosters wider collaboration and increased citations

- maximum visibility for your research: over $100 \mathrm{M}$ website views per year

At $\mathrm{BMC}$, research is always in progress.

Learn more biomedcentral.com/submissions 